\title{
Corporate Social Responsibility (CSR) und Corporate Citizenship (CC): Selbstbild und Fremdwahrnehmung in der öffentlichen Kommunikation
}

\author{
Voraussetzung, Strukturen und Formen \\ nachhaltigkeitsorientierter Verantwortungs- \\ kommunikation am Beispiel der Medienwirtschaft
}

Isabel Bracker

\section{Zusammenfassung}

Medienunternehmen standen bislang selten im Fokus der CSR-Forschung. In diesem Forschungsprojekt wurden sie auf gleich drei Ebenen analysiert: Neben der Wahrnehmung gesellschaftlicher Verantwortung wurde ebenso die öffentliche Kommunikation über Verantwortung einbezogen, was durch die Analyse von strategischer Kommunikation der Medienunternehmen über ihre Aktivitäten geschah sowie durch die Untersuchung der journalistischen Berichterstattung über diese. Dabei wurde ein ganzheitlicher Ansatz gewählt und neben der Wahrnehmung gesellschaftlicher Verantwortung, gleichsam die Vernachlässigung bzw. unverantwortliches Handeln (Corporate Social Irresponsibility) einbezogen. Einige Ergebnisse werden im nachfolgenden Beitrag präsentiert. Als theoretische Basis diente die Strukturationstheorie von Giddens, durch deren Anwendung ein Framework erarbeitet werden konnte, der zudem als übertragbar und als höchst anschlussfähig erachtet wird.

\section{Gesellschaftliche Verantwortung von Medienunternehmen}

Zwar ist die gesellschaftliche Verantwortung von Unternehmen und ihr bürgerschaftliches Engagement in den letzten Jahren vermehrt in den Fokus von Öffentlichkeit und Wissenschaft gerückt (Schmidpeter, 2012: 1; Palazzo, 2009: 2). Medienunterneh-

(C) Der/die Autor(en) 2017

K.-D. Altmeppen et al. (Hrsg.), Nachhaltigkeit in Umwelt,

Wirtschaft und Gesellschaft, DOI 10.1007/978-3-658-14439-5_11 
men standen dabei bislang jedoch selten im Zentrum. Dies kann zum einen darauf zurückgeführt werden, dass der Medienbranche im Vergleich zu anderen Branchen nachgesagt wird, nur einen kleinen ökologischen Fußabdruck zu hinterlassen (Gulyás, 2009: 659; Toennesen et al., 2013: 9). Lauter sind die Stimmen da bereits hinsichtlich der sozialen Dimension, vor allem in Hinblick auf die Arbeitsbedingungen der Mitarbeiter (Altmeppen, 2008a: 247). Zum anderen könnte dies aber auch darauf hindeuten, dass noch zu wenig Material vorlag, das untersucht werden konnte.

Hinzu kommt, dass Medienunternehmen häufig ganz allgemein mit den von ihnen distribuierten Inhalten assoziiert werden (Hohlfeld, 2002: 101) und ihnen deshalb Verantwortung stark in Bezug auf die Inhalte zugeschrieben wird (Hülsewiesche, 2010; Toennesen et al., 2013). Auch in wissenschaftlichen Betrachtungen wurde dies häufig vermengt betrachtet und damit - im Grunde - häufig die Verantwortung der Journalisten, nicht die der Medien als Wirtschaftsakteur, analysiert. ${ }^{1}$

Dass beide Ebenen eng verwoben sind, ist unbestritten (Gulyás, 2009: 659). Die Intensität zeigt sich z. B. bei Axel Springer, wenn das Unternehmen formuliert, dass es sich nicht nur aus gesellschaftlicher Verantwortung „für ein nachhaltiges Wirtschaften, die Sicherung sozialer und ökologischer Standards sowie für die Gesellschaft als Ganzes" engagiere, sondern auch der journalistischen Glaubwürdigkeit wegen, denn, „[w]er sich kritischem Journalismus verpflichtet, muss selbst einer genauen Überprüfung standhalten" (Axel Springer, 2015; Hervorheb. im Original; Anpassung I.B.). Wer also symbolisch mit Steinen werfen will (ergo die Verantwortung anderer kritisiert), sollte nicht im Glashaus sitzen.

Dass beide Ebenen unterschieden und mit ihnen Aufgaben und folglich auch Verantwortungsbereiche differenziert werden können und sollten (Altmeppen, 2006, 2011a), wird in diesem Beitrag als Fundament der weiteren Überlegungen zu Corporate Social Responsibility (CSR) und Corporate Citizenship (CC) von Medienunternehmen dienen. Neben dieser Differenzierung (siehe dazu Abschnitt 2.2.) ist es die Strukturationstheorie von Anthony Giddens (1997; Abschnitt 2.3), die als theoretische Basis dient, sowie dazu, CSR und CC empirisch handhabbar zu machen (zu Methoden, Instrumenten und Ergebnissen s. Abschnitt 3 und 4).

Im Fokus des Projektes standen die Fragen, wie gesellschaftliche Verantwortung (CSR/CC) in ausgewählten Medienunternehmen wahrgenommen wird, wie die Unternehmen selbst über ihre gesellschaftliche Verantwortung öffentlich kommunizieren (Kommunikation von Verantwortung, Selbstbild) und welches Fremdbild in der journalistischen Berichterstattung darüber geschaffen wurde (Kommunikation über Verantwortung).

1 Dies zeigt sich bei der Auswertung bislang zum Thema erstellter Studien (Abschnitt 2.4). 
Zunächst werden die Grundlagen und Zusammenhänge in Bezug auf Verantwortung, CSR, CC und Nachhaltigkeit gelegt (Abschnitt 2.1).

\section{Theoretische Grundlagen}

\subsection{Unternehmerische Verantwortung und Nachhaltigkeit}

Verantwortung wird „als die zentrale Größe“ angesehen, „über die sich Nachhaltigkeit“ durch (und in) Unternehmen „realisieren lässt“ (Karmasin \& Weder, 2008: 66). ${ }^{2}$ Grunwald \& Kopfmüller (2012: 11) folgend wird unter Nachhaltigkeit der Zustand verstanden, der am Ende des gesellschaftlichen Wandlungsprozesses, der nachhaltigen Entwicklung, steht. Diese wird „(n)ach der heute überwiegend akzeptierten Definition“ dann als „realisiert“ angesehen, „wenn (...), die Bedürfnisse der Gegenwart befriedigt ““ werden können (Grunwald \& Kopfmüller, 2012: 11), „,ohne zu riskieren, dass künftige Generationen ihre eigenen Bedürfnisse nicht befriedigen können' (Hauff 1987: 46)“ (Grunwald \& Kopfmüller, 2012: 11; Anpassung I.B.). Nachhaltigkeit wird in dieser Arbeit ähnlich wie von Jonker et al. (2011: 9) als Rahmen verstanden, der auch die Fragen und Diskussionen um den Beitrag der Medienunternehmen zu einer nachhaltigen Entwicklung umfasst. Medienunternehmen werden als Teil des Gesamtsystems (gesamtgesellschaftliche Ebene) verstanden, die sich auf einzelwirtschaftlicher Ebene (Ebene des Unternehmens) (gesellschaftlich) verantwortlich zeigen müssen, um ihren Beitrag zur Erreichung des Idealzustands (Nachhaltigkeit) zu leisten (u. a. Mast \& Stehle, 2009: 174; Loew et al., 2004: 72; Grunwald \& Kopfmüller, 2012: 184). Es wird davon ausgegangen, dass Unternehmen sowohl positive als auch negative Auswirkungen auf ihre Umwelt und die Gesellschaft haben. Im Sinne des gesellschaftlich verantwortlichen Handelns müssen sie die negativen Auswirkungen verringern und die positiven verstärken (Zyglidopoulos et al., 2012; Grayson, 2009: 6, 8).

Wird Verantwortung als Zuschreibungsbegriff verwendet, kann er in mehrere Relationen zerlegt werden (Werner, 2006: 543), wodurch die Frage nach der Wahrnehmung und Kommunikation von Verantwortung durch Medienunterneh-

2 Die Zusammenhänge und die Annäherung der Konzepte ,Nachhaltigkeit' und , unternehmerische Verantwortung' wurden von Schneider (2012: 20, 25ff.) herausgearbeitet. Als verbindendes Element wird die „triple bottom line“ angesehen (u.a Schneider, 2012: 24f.; Jarolimek, 2012: 136; Jarolimek \& Raupp, 2011: 16f.), denn sowohl Nachhaltigkeit als auch unternehmerische Verantwortung beziehen sich auf dieselbe Basis: eine soziale, ökonomische und ökologische Dimension (Jarolimek \& Raupp, 2011: 16f.). 
men handhabbar wird. Denn dann schließt der Verantwortungsbegriff, als „ein zuschreibungsgebundener mehrstelliger Relations- bzw. Strukturbegriff“" und als „interpretations- und analysebedürftiges Schema“" (Maring, 2001: 13f.), nach Maring (2001: 14) folgende Elemente ein:

- jemand: Verantwortungssubjekt, -träger (Person, Korporationen) ist

- für: etwas (Handlungen, Handlungsfolgen, Zustände, Aufgaben usw.)

- gegenüber: einem Adressaten

- vor: einer (Sanktions-, Urteils-) Instanz

- in bezug auf: ein (präskriptives, normatives) Kriterium bzw. Werte, Maßstäbe

- im Rahmen eines: Verantwortungs-, Handlungsbereiches verantwortlich.

Angewendet auf das Forschungsprojekt bedeutet dies, dass zunächst das Verantwortungssubjekt konkretisiert werden muss: Im Zentrum steht, wie Medienunternehmen für ihre Handlungen, Handlungsfolgen, Zustände, Aufgaben usw. (Verantwortungsobjekt) gegenüber jemandem/etwas (Adressaten) Verantwortung übernehmen.

Um die Frage, ob Unternehmen allerdings wie Individuen Verantwortung zugeschrieben werden kann, entfaltete sich eine komplexe Debatte. So geht Giddens (1997: 278) grundsätzlich davon aus, dass „allein Individuen, Wesen, denen eine körperliche Existenz zukommt" Handelnde sind. Organisationen jedoch kann ein Akteurstatus eingeräumt werden, „wenn ein bedeutsames Maß an reflexiver Steuerung der Bedingungen der sozialen Reproduktion gegeben ist" (Giddens, 1997: 278). Organisationen erfüllen diese Bedingung bspw. durch „rekursiv organisierte Regeln und Ressourcen und meist implizite Orientierungsmuster (z. B. Normen, Rollengefüge, internen Kommunikationsstrukturen)" (Röttger, 2010: 134). Die Organisation erlangt somit eine „spezifische ,Identität" (Röttger, 2010: 134; mit Verweis auf Zerfaß, 1996: 94f.), und kann als organisierter Akteur bzw. korporativer Akteur gefasst werden (Röttger, 2010: 134). Es bleibt jedoch eine „kürzelhafte Darstellung“ (Giddens, 1997: 279), denn letztlich sind es nicht die Organisationen, die handeln und in Folge Verantwortung tragen, da „hinter dem ,Handeln der Organisation immer das Handeln individueller Akteure steht" (Röttger, 2010: 134; mit Verweis auf Schneidewind, 1998: 42f. und Giddens, 1997: 278f.).

Corporate Social Responsibility ${ }^{3}$ und Corporate Citizenship sind in der Kommunikationswissenschaft die hauptsächlich gebräuchlichen Begriffe, um die spezielle, aber generell auf Unternehmen als Akteure bezogene Verantwortung zu beschreiben (Altmeppen \& Bracker, im Druck). Verantwortung ist der Dachbegriff, CSR und

3 „Social“ wird hier nicht eng als „sozial“ übersetzt, sondern meint die gesellschaftliche Verantwortung („social“ = societal/gesellschaftlich) der Unternehmen. 
CC werden „als Ableitungen von Verantwortung mit spezifischen Merkmalen definiert“ (Altmeppen \& Bracker, im Druck; Altmeppen, 2011a: 248).

Was aber wird unter CSR und CC verstanden? Darüber besteht kein Konsens (u. a. Schaltegger, 2011: 188). Als Grundstein der Diskussion über die gesellschaftliche Verantwortung von Unternehmen unter dem Begriff CSR wird jedoch meistens die Arbeit von Howard R. Bowen (1953) ${ }^{4}$ herangezogen (Carroll, 1999: 269), außerdem können sich wiederholende CSR/CC ${ }^{5}$ konstituierende Elemente ausgemacht werden. Aus diesen wurde im Rahmen der Arbeit eine Matrix zusammengestellt, die die wesentlichen Merkmale enthält und das Spektrum, das nachfolgend näher erläutert wird, abbildet. Denn wie auch bereits von Galonska (2012: 289) beschrieben, geht es weniger darum, „eine abschließende und allumfassende Definition“ zu liefern als um einen „Arbeitsbegriff“ bzw. hier einen Rahmen „zum Zweck empirischer Analysen“" (Galonska, 2012: 289) zu entwickeln. ${ }^{6}$

Nach Raupp et al. (2011: 10ff.) kann zwischen einem engen und einem weiten/ umfassenden Verständnis von CSR unterschieden werden und somit ein Spektrum aufgespannt werden. Im weiten Verständnis wird auch „die Gewinnmaximierung oder die Einhaltung gültigen Rechts (,Compliance')“ als CSR anerkannt, wodurch viele Aktivitäten als CSR bezeichnet werden können (Raupp et al., 2011: 11; Carroll, 1979; Hiß, 2007). Dem engen Verständnis nach muss CSR a) mit dem Kerngeschäft des Unternehmens in Verbindung stehen, b) freiwillig geschehen und c) auf die Nachhaltigkeit der Ressourcen ausgerichtet sein (Raupp et al., 2011: 11), was bedeutet, dass „nicht bereits durch eine reine Gewinnerzielung“ ökonomische Verantwortung wahrgenommen wird, „sondern erst dann, wenn die Gewinne ökonomisch verantwortlich für nachhaltige, d.h. zukunftssichernde Maßnahmen eingesetzt werden“ (Raupp et al., 2011: 12). Variabel ist demnach bereits der Punkt, an dem CSR beginnt.

4 Er wird als „Urvater“ und Begriffsschöpfer bezeichnet (Carroll, 1999: 270), was aber nicht bedeutet, dass die unter CSR summierten Inhalte nicht schon früher diskutiert wurden (u. a. Carroll, 1999; Schaltegger, 2011: 187). Doch finden sich unterschiedliche Herangehensweisen, oft wird der Begriff „missverständlich und widersprüchlich genutzt“ (Schaltegger, 2011: 188) oder mit verwandten Begriffen wie Corporate Citizenship oder Corporate Sustainability synonym verwendet (Schaltegger, 2011: 188; zur Differenzierung Schaltegger, 2011: 189, 193ff.). Für einen Überblick über verschiedene Ansätze und Theorien zu CSR/CC siehe Garriga \& Melé (2004), die eine Einordnung in ethische, politische, instrumentalistische und integrative Theorien/Konzepte vornahmen.

5 Galonska et al. (2007) folgend wird es in diesem Beitrag als sinnvoll erachtet, CSR und CC meinend von "gesellschaftlicher Verantwortung" der Unternehmen (oder verkürzt CSR) zu sprechen und eine genauere Abgrenzung nur vorzunehmen, wenn sie sinnvoll ist (auch Bluhm, 2008: 144).

6 Dies wird z. T. auch als gar „nicht möglich“ oder sogar als „kontraproduktiv“ aufgefasst, da CSR von der „kontinuierlichen Entwicklung“lebe (Schneider, 2012: 19). 
An dieser Unschärfe setzen Tench et al. (2012: 8ff.) an, deren Ansatz es ist, dass die gesellschaftliche Verantwortung von Unternehmen ohne Corporate Social Irresponsibility (CSI) nicht richtig er- und gefasst werden kann. CSI dagegen markiert einen klaren Startpunkt, ab dem die Handlungen der Unternehmen skaliert und verortet werden können (Tench et al., 2012: 8; Clark \& Grantham, 2012). Mit dieser Grundlage kann zunächst entschieden werden, ob es sich um Aktivitäten handelt, die illegal sind. Darauf folgen Abstufungen, in denen hochgradig unnachhaltiges/ unethisches Handeln (das gesellschaftlich inakzeptabel ist) von solchen Aktivitäten unterschieden wird, bei denen geringfügig unnachhaltiges/unethisches Handeln (bei dem fraglich ist, ob es gesellschaftlich akzeptabel ist) vorliegt (Tench et al., 2012: 8f.). Es folgen weitere Stufen bis hin zu nachhaltigem/ethischem Handeln, das als gesellschaftlich akzeptabel eingestuft werden kann (Tench et al., 2012: 9).

Auch Imbusch und Rucht (2007) haben sich in dieser Richtung mit Verantwortung von Unternehmen auseinandergesetzt. Ihr "Klassifikationsschema“ (Galonska et al., 2007: 20) umfasst acht Stufen von der „kategorischen Verweigerung bzw. Verletzung von Verantwortlichkeit" bis hin zu sehr engagiertem und verantwortlichem Handeln (Galonska et al., 2007: 17f.; s. auch die einzelnen Stufen in Tabelle 2). ${ }^{7}$

Das Spektrum von CSI bis CSR kann demnach mit den Stufen von Galonska et al. (2007) konkretisiert werden (s. Tabelle 1 und Tabelle 2) und es können illegale Handlungen (die gesellschaftlich nicht akzeptabel sind, z. B. wenn aus Eigennutz eine Regel verletzt wird (Stufe 1)) von hochgradig unnachhaltigen/unethischen unterschieden werden (denn alles was legal ist, muss nicht verantwortungsvoll sein; Stufe 2). Weiterhin können Handlungen differenziert werden, bei denen es fraglich ist, ob sie gesellschaftlich akzeptabel sind (sie werden als geringgradig unnachhaltig/unethisch eingeordnet). Unter „weite CSR" können Handlungen (z. B. Gesetzeseinhaltungen oder Gewinnmaximierung; Stufen 3-5) von denjenigen unterschieden werden, die als „,enge CSR“ gelten, die freiwillig durchgeführt werden und nach der Definition von Galonska et al. (2007: 13) als gesellschaftliche Verantwortung anzusehen sind (Stufen 6-7). Denkbar sind verantwortliche bzw. verantwortungslose Handlungen auf allen Dimensionen: ökonomisch, ökologisch, sozial, aber auch kommunikativ ${ }^{8}$ und als kontextspezifische Erweiterung auch medial. ${ }^{9}$

7 Fallbeispiele werden in Imbusch und Rucht (2007) detailreich beschrieben.

8 Die kommunikative Dimension wurde in Anlehnung an Karmasin und Weder $(2008,2009)$ einbezogen. Darunter werden Verantwortungwahrnehmungen gefasst, die sich maßgeblich auf eine kommunikative Ebene beziehen, z. B. Stakeholder-Dialoge, das Schaffen von Transparenz, z. B. in Zusammenhang mit Instrumenten wie Nachhaltigkeitsberichten, aber auch verantwortungsvolle interne Kommunikation, womit eng die Frage verbunden ist, ob die Art und Weise, wie kommuniziert wird, verantwortungsvoll ist.

9 Die mediale Dimension bezieht sich auf Verantwortungswahrnehmungen, die „on air“ geschehen. Im Unterschied zu anderen Branchen nutzen Medienunternehmen ihre 


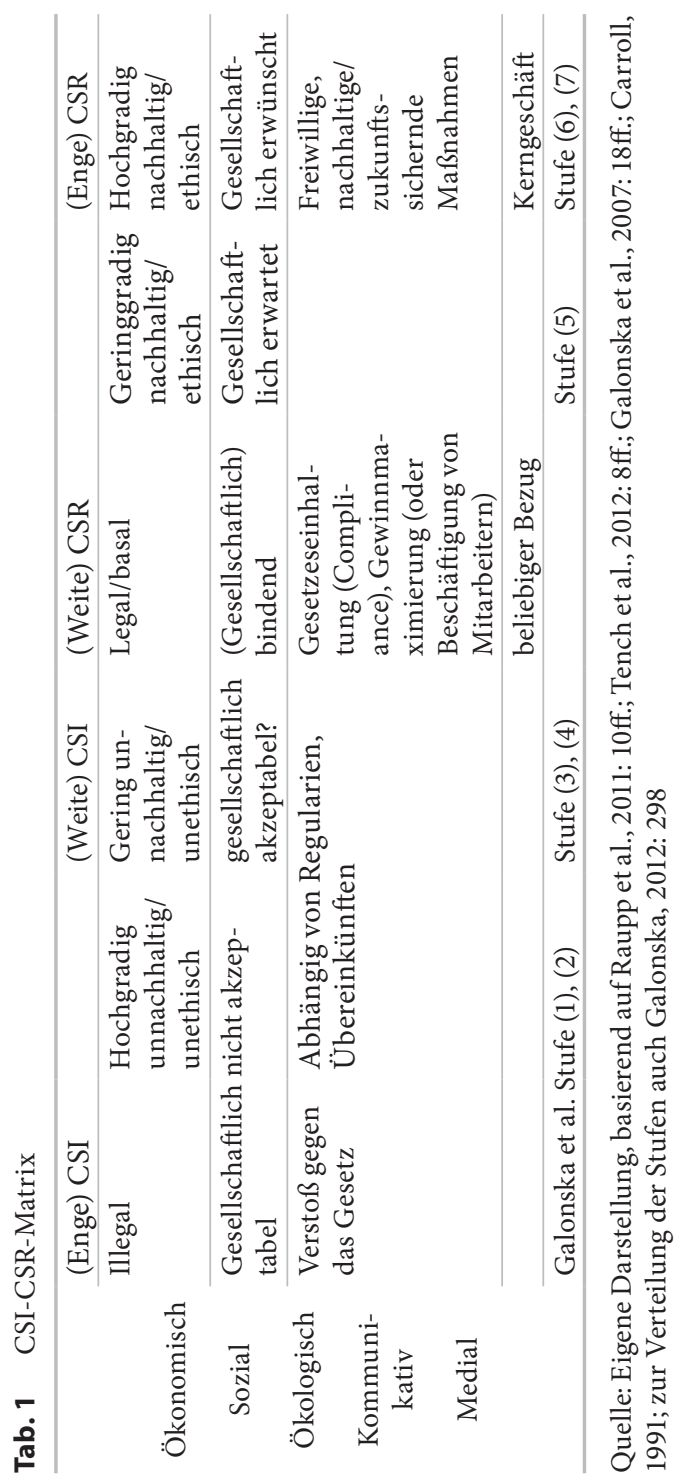

Reichweite, um z. B. Botschaften von sozialen Organisationen zu verbreiten (und stellen Sendezeit kostenfrei zur Verfügung). Hierunter fallen aber auch Spendenaufforderungen und Spendengalas, die übertragen werden. 


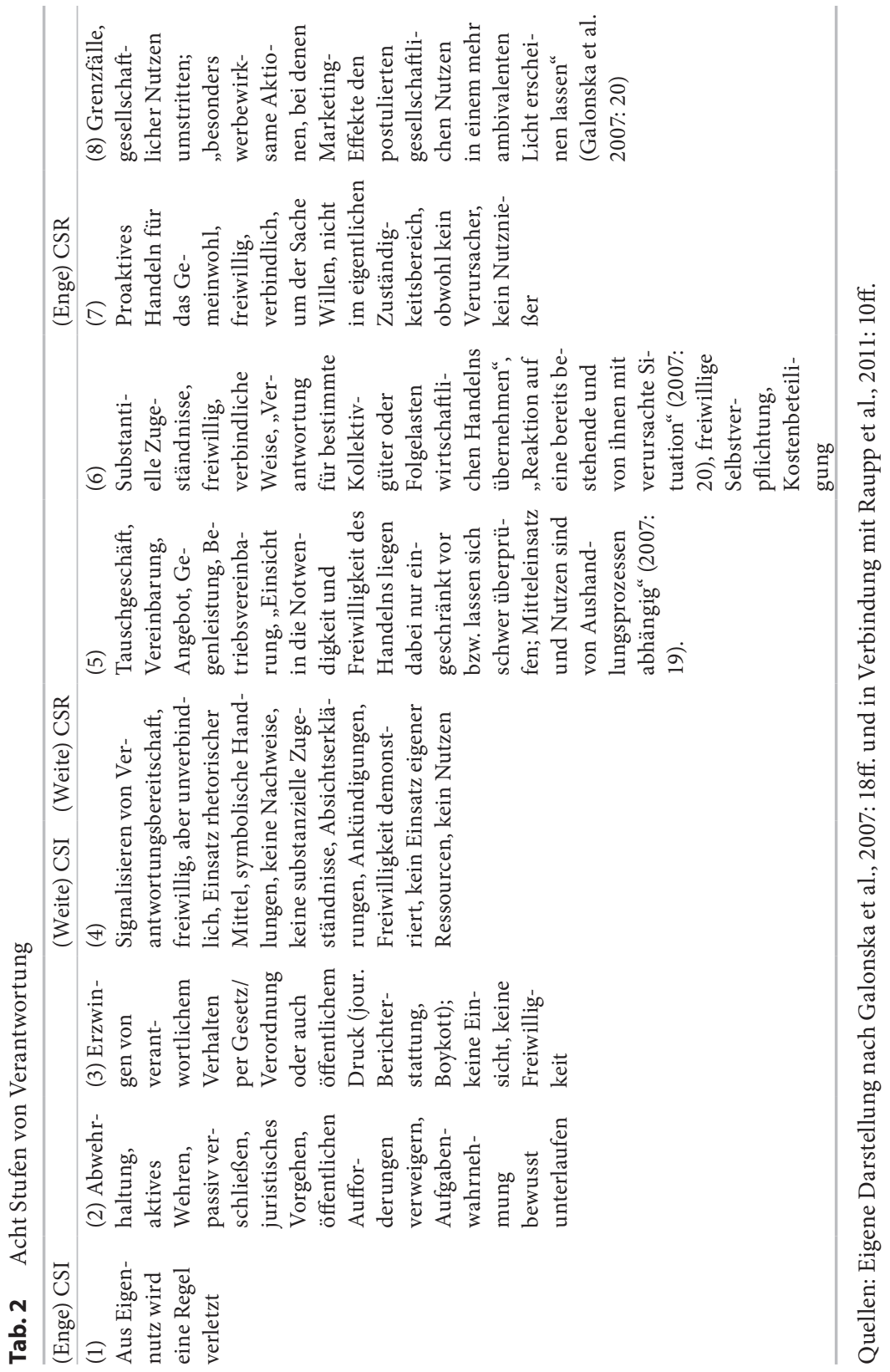


Der nächste Abschnitt widmet sich der Konkretisierung von Medienunternehmen, ihren Aufgaben und Verantwortungen.

\subsection{Medienunternehmen, ihre Aufgaben und Verantwortungen}

Grundlegend für das Forschungsprojekt war eine differenzierte Beschäftigung mit Medienunternehmen. Diese mussten nicht nur definiert und somit abgrenzbar gemacht werden - nicht zuletzt in Hinblick auf das empirische Vorgehen -, es bedurfte auch einer genauen Betrachtung der Abläufe, Aufgaben und Verantwortungen innerhalb der Medienunternehmen, um die gesellschaftliche Verantwortung näher bestimmen und davon ausgehend die eigenen Zuschreibungen der Medienunternehmen und der von Dritten einordnen zu können.

Darum musste zunächst geklärt werden, was unter Medienunternehmen verstanden wird. ${ }^{10}$ Es zeigt sich, dass nicht viele Begriffsbestimmungen vorhanden sind (Altmeppen, 2006: 12) und zudem, dass unter den existierenden kein Konsens besteht (Altmeppen, 2011b: 233; Altmeppen, 2006: 153ff.; Weber \& Rager, 2006: 119f.) und zugleich ein breites Spektrum abgedeckt wird. Dieses reicht von solchen Definitionen, die unter Medienkonzernen Unternehmen verstehen, „die sich als solche bezeichnen beziehungsweise von anderen als solche eingeschätzt werden“ (Kleinsteuber \& Thomaß, 2004: 126) bis hin zu solchen, die Medienunternehmen erst als solche bezeichnen, wenn sie „ihren wirtschaftlichen Schwerpunkt in der aktuellen/journalistischen Produktion von Informationen haben“" (Heinrich, 2010: 27).

Eine sehr differenzierte Definition wird von Wirtz (2013) geliefert. Medienunternehmen werden dort „als planvoll organisierte Wirtschaftseinheiten“ definiert, die „eigen- und fremderstellte[.] redaktionelle[.] Inhalte (informatorische und/oder unterhaltende Inhalte)“ bündeln, diese „auf ein speicherfähiges Trägermedium“ transformieren und „die direkte oder indirekte Distribution“ durchführen (Wirtz, 2013: 16, Anpassungen I.B.). Danach sind z. B. Fernseh- und Radiosender, Zeitungsund Zeitschriftenverlage sowie Buchverlage Medienunternehmen, Druckereien und Speichermedienhersteller jedoch keine (Wirtz, 2013: 12).

Ferner soll, Altmeppen (2006: 263) folgend, zwischen zwei Organisationseinheiten der Medienunternehmen unterschieden werden: Den Medien (als Organisation) und der journalistischen Organisation. Sie stellen „eigenständige, funktional und strukturell unterscheidbare Organisationseinheiten“ dar (Altmeppen, 2011a:

10 Die Begriffe Medienunternehmen und -organisationen werden oftmals synonym verwendet (Altmeppen 2006: 153). 
248), deren „wesentliche Unterscheidungsmerkmale "ihr Orientierungshorizont, ihre institutionelle Ordnungen und ihre Akteurkonstellationen sind (Altmeppen, 2006: 263). Der Journalismus agiert „im Orientierungshorizont der Öffentlichkeit“ (Altmeppen, 2006: 108), „die Medien dagegen im Orientierungshorizont der Wirtschaft" (Altmeppen, 2006: 263). Dies bedeutet, dass der Journalismus „nach der Referenz von öffentlich/nicht-öffentlich“ "handelt, die Medien hingegen „betreiben ein Geschäft und handeln dabei ökonomisch, nach der Referenz von Zahlung/ Nichtzahlung" (Altmeppen, 2011a: 249). Diese Codes (öffentlich/nicht-öffentlich und zahlen/nicht-zahlen) sichern ,in beiden Organisationen die Richtigkeit des Handelns der Organisationsmitglieder - und insbesondere ihrer Entscheidungen - anhand einer Leitdifferenz" (Altmeppen, 2006: 202).

Auch die institutionellen Ordnungen unterscheiden sich: Der Journalismus produziert aktuelle „Informationsangebote“ (sie umfassen „Information, Infotainment, Unterhaltungsjournalismus“ (Altmeppen, 2006: 109)), seine Funktion liegt in der Herstellung öffentlicher Kommunikation, die Medien hingegen bündeln ${ }^{11}$ Angebote zu „spezifischen Produktgruppen“ (Altmeppen, 2006: 110), die neben Journalismus auch Unterhaltung und Werbung beinhalten, um sie schließlich zu distribuieren. ${ }^{12}$ Durch die Distribution stellen sie die „Finanzierung der Medienangebote“ (Altmeppen, 2006: 263) sicher und „Zahlen“13 für „die Zulieferung von Inhalten (...) und statten den Journalismus mit Ressourcen aus" (Altmeppen, 2011a: 249). Beiden Einheiten fehlt die Möglichkeit, die Aufgabe des anderen zu übernehmen (Altmeppen, 2006: 264), sie stehen auf der „organisationalen Ebene (...) in enger Beziehung zueinander" (Altmeppen, 2006: 264) und kooperieren in Ko-Orientierung (Altmeppen, 2011a: 248). Bezüglich der Akteurkonstellation kann zwischen Redaktions- und Medienmanagement unterschieden werden und damit zwischen verschiedenen Akteuren, die unterschiedliche Ziele verfolgen und Aufgaben ausführen (Altmeppen, 2006: 254).

Abgeleitet bedeutet dies, dass der Journalismus für die Inhalte verantwortlich ist und die journalistische Verantwortung trägt (Altmeppen, 2011a: 257). Hingegen

11 Ausnahmen zeigen sich bei Printmedien, wenn die Planung und die Bündelung (der redaktionellen Teile) an die Redaktionen „delegiert" wurde (Altmeppen, 2006: 160, 215; 2008b: 87).

12 Erst durch die Distribution wird aus Unikaten (zunächst ist jede Nachricht, jeder Unterhaltungsbeitrag ein Unikat) eine kommerzielle, massenhafte Ware, „nur sie erfolgt massenhaft" (Altmeppen, 2008c: 240; 2006: 148)

13 Bezahlen ist als Metapher zu verstehen, gemeint ist die Ressourcenausstattung des Journalismus durch die Medien (Tausch von Informationsprodukten gegen Ressourcen, was aber u.a. die Bezahlung des journalistischen Personals einschließt) (Altmeppen, 2006: 204). 
bezieht sich die Verantwortung der Medien auf die Ressourcensicherung für den Journalismus (diese Ebene unterscheidet die Medienunternehmen von anderen Unternehmen, deren Produkte nicht an einen gesellschaftlichen Auftrag gekoppelt sind; die über die Grundsicherung hinausgehende Verantwortung wird auch als „Media Social Responsibility“ bezeichnet (Altmeppen, 2011a: 264)) sowie als Wirtschaftsakteur auf die Gesellschaft (Altmeppen, 2011a: 258). Diese Verantwortung und Ebene unterscheidet sich nicht von der gesellschaftlichen Verantwortung anderer Unternehmen.

Diese Differenzierung wird in Wissenschaft und Praxis selten getroffen und somit wurden "CSR activities, which exceed the democratic mission of media organizations (...) hardly been adressed in research so far" (Ingenhoff \& Koelling, 2012: 156).

\subsection{CSR aus strukturationstheoretischer Perspektive}

In diesem Abschnitt soll auf der Grundlage der organisationalen Unterscheidung von Journalismus und Medien die gesellschaftliche Verantwortung aus strukturationstheoretischer Perspektive beleuchtet werden. Mit der Strukturationstheorie gelingt es, den Dualismus zwischen System- und Handlungstheorie zu überwinden (Klare, 2010: 91), da Giddens (1997: 77) von der „Dualität von Struktur“ ausgeht. Struktur wird als "rekursiv organisierte Menge von Regeln und Ressourcen“ begriffen (Giddens, 1997: 77), d. h. zum einen, dass Strukturen Handeln ermöglichen, sie es aber auch begrenzen; zum anderen, dass in und durch Handlungen Strukturen reproduziert, sie aber im Handeln auch modifiziert und (neu) produziert werden (Giddens, 1997: 77f.). Handlung und Struktur stehen sich demnach „nicht mehr konkurrierend gegenüber, sondern bedingen sich gegenseitig“" (Klare, 2010: 92, Hervorheb. im Orig.; Lamla, 2003: 45).

Der Projektarbeit liegt nicht nur die Annahme der Rekursivität zugrunde, sondern auch die der Reflexivität, was bedeutet, dass die handelnden Akteure - die für CSR verantwortlichen Personen der Medienunternehmen - grundsätzlich nicht nur Auskunft darüber geben können, welche Aktivitäten und Maßnahmen in diesem Bereich von ihnen durchgeführt oder angestoßen werden, sondern auch, warum sie auf welche Weise handeln und worauf (auf welche Strukturen) sie sich dabei beziehen. ${ }^{14}$ Gleiches gilt für die Kommunikation über diese Aktivitäten. Die in den

14 Denn neben dem praktischen Bewusstsein wird den Akteuren auch ein diskursives Bewusstsein zugesprochen, d.h. „kompetente Akteure“ können „die Absichten ihres Handelns und dessen Gründe fast immer diskursiv darlegen“ (Giddens, 1997: 57), sie sind „normalerweise dazu in der Lage (...), für ihr Handeln in aller Regel eine Erklärung 
Unternehmen zu CSR vorhandenen Regeln und eingesetzten Ressourcen liefern letztlich Hinweise auf die Verbindlichkeit und das Ausmaß der Wahrnehmung von Verantwortung.

Innerhalb der ausgewählten Medienunternehmen wurde ausgehend von Giddens (1997) (analytisch) zwischen der Handlungsebene und der Strukturebene unterschieden: Auf der Handlungsebene sind die Akteure tätig, die die CSR-/CC-Aktivitäten und -Maßnahmen konzipieren und umsetzen (s. dazu Kapitel 3). Gleiches gilt für die Kommunikation der Wahrnehmung. Auch hier gibt es Akteure, die die Kommunikation der CSR-/CC-Aktivitäten und -Maßnahmen planen und durchführen. Weiterhin existieren auf der Strukturebene in den Unternehmen Regeln und Ressourcen, die die Handlungen der Akteure ermöglichen und begrenzen, die von den Akteuren reproduziert, aber auch (neu) produziert werden.

Definiert werden „Regeln des gesellschaftlichen Lebens als Techniken oder verallgemeinerbare Verfahren (...), die in der Ausführung/Reproduktion sozialer Praktiken angewendet werden“" (Giddens, 1997: 73). Sie "beziehen sich einerseits auf die Konstitution von Sinn und zum anderen auf die Sanktionierung sozialer Verhaltensweisen“ (Giddens, 1997: 70, Hervorheb. im Orig.), d.h. es geht erstens um „Deutungsregeln“ (die dem Handeln einen Sinn geben), „die als Interpretationsschemata die Konstitution von Sinn ermöglichen", auf die sich die Akteure beim Handeln in ihrer Kommunikation beziehen (Schwarz, 2008: 66) und zweitens um Regeln, die „die Sanktionierung sozialer Verhaltensweisen“ betreffen, „so daß unter Bezugnahme auf Normen eine Rechtfertigung einer Handlung möglich ist bzw. die Missachtung von Normen sanktioniert werden kann"(Schwarz, 2008: 66).

Daneben werden Ressourcen als Teil der Struktur angesehen, unterschieden nach autoritativen und allokativen Ressourcen (Giddens, 1997: 81, 86). Allokative Ressourcen werden als „[a]n der Generierung von Macht beteiligte materielle Ressourcen einschließlich der natürlichen Umwelt und physischer Artefakte" verstanden, sie „leiten sich aus der Herrschaft des Menschen über die Natur her" (Giddens, 1997: 429, Anpassung I.B.). Autoritative Ressourcen hingegen sind „[a]n der Generierung von Macht beteiligte nichtmaterielle Ressourcen, die sich aus dem Vermögen, die Aktivitäten menschlicher Wesen verfügbar zu machen, herleiten“, sie „ergeben sich aus der Herrschaft von Akteuren über andere Akteure“ (Giddens, 1997: 429, Anpassung I.B.).

Regeln und Ressourcen, die Strukturmodalitäten, können als „Bindeglied“ (Klare, 2010: 99, Hervorheb. im Orig.) zwischen Struktur und Handlung verstanden werden: „Interaktionen (soziale Handlungen) beinhalten demnach die Kommu-

abzugeben, wenn sie danach gefragt werden“ (Giddens, 1997: 56), daher können die Absichten grundsätzlich durch Experteninterviews erfragt werden. 
nikation von Sinn und Bedeutung (auf Basis der Deutungsregeln), die Ausübung von Macht durch Anwendung von Ressourcen und Sanktion mittels regulativer Regeln (Handlungsregeln)“ (Klare, 2010: 100). ${ }^{15}$

Mithilfe der Strukturationstheorie wurden Regeln und Ressourcen innerhalb der Medienunternehmen in Bezug auf CSR/CC identifiziert (und in den Instrumenten operationalisiert). Wird CSR in einem Unternehmen ernst genommen, wird das ganze Unternehmen davon geprägt. CSR-typische Handlungen werden ermöglicht, sie können durch die Bezugnahme auf CSR legitimiert und durchgesetzt werden, da CSR ein Machtmittel darstellen kann. Auch können neue Räume, Handlungsfelder und Märkte erschlossen werden, etwa wenn beispielsweise Produktmodifikationen/-innovationen vorgenommen werden, die erst durch die Integration von CSR angestoßen wurden. Auch Wettbewerbsvorteile können entstehen, wenn CSR ein Alleinstellungsmerkmal in der Branche darstellt. CSR begrenzt das Unternehmen bzw. seine Akteure aber auch, denn Verantwortung - ernst genommen - muss bestimmte Handlungen unmöglich machen, z. B. den Einkauf von billigeren Waren, deren Unbedenklichkeit nicht ausreichend geklärt ist.

Als Signifikationsregel kann die Unternehmensmission/-vision identifiziert werden (Röttger, 2005: 14; Ortmann et al., 1990: 30; Röttger, 2010: 138; Becker, 1996: 141, 194), sie leitet die Handlungen der CSR-Verantwortlichen, denn sie müssen die Aktivitäten in Bezug zu diesen vorgegebenen Leitbildern reflektieren und ggf. anpassen. Dies gilt auch für die CSR-Definition des Unternehmens oder das Ziel, das damit verbunden ist. Beide sind handlungsleitend, befähigen dazu, Entscheidungen zu treffen und können als Mittel dienen, um Mitarbeiter zu überzeugen, sich $\mathrm{zu}$ engagieren. Gleichsam können, durch die Interpretation der verantwortlichen Akteure, die Leitbilder modifiziert und das Verständnis von CSR verändert werden.

Als Legitimationsregeln gelten bspw. Gesetze, aber auch organisationsinterne Normen und Regeln wie auch informelle Leitlinien. Weiterhin stehen durch die organisationale/hierarchische Struktur Ressourcen zur Verfügung, dies können nicht nur physische (allokative) wie Personal, Budget oder Rohstoffe sein, die eingesetzt werden, um CSR-Aktivitäten umzusetzen, sondern auch autoritative Ressourcen. Die häufig enge Angliederung von CSR-Verantwortlichen beim Vorstand oder die direkte Zuordnung der Thematik beim Geschäftsführer verleiht CSR und ihren Akteuren Durchsetzungskraft. Auch informelle Ressourcen wie Vernetzung,

15 In den meisten Interpretationen von Giddens (1997) werden Regeln auf Signifikation und Legitimation und Ressourcen auf Domination bezogen. Dies kann jedoch auch weitergedacht werden und alle Dimensionen können sowohl auf Regeln als auch Ressourcen bezogen werden (Bracker, Schuhknecht \& Altmeppen im Druck). Auch Ortmann et al. (1990: 24ff.) betonen die Rolle der Domination und der Macht. 
Glaubwürdigkeit und Anerkennung des Verantwortlichen (individuelle Ressourcen, Röttger, 2010: 166) können eine fördernde oder hemmende Rolle haben.

\subsection{Forschungsstand CSR und Medienunternehmen}

Im deutsch- und englischsprachigen Raum konnten nur wenige Arbeiten recherchiert werden, die Medienunternehmen und ihre gesellschaftliche Verantwortung (empirisch) analysierten. Ein intensiverer Blick darauf zeigt, dass sich diese vornehmlich mit der Analyse von CSR in der öffentlichen Kommunikation der Medienunternehmen beschäftigen (also mit dem Selbstbild). Hauptsächlich wurden Geschäfts-/ Jahres-/CSR-Berichte und/oder Websites/Online-Informationen der Unternehmen inhaltsanalytisch untersucht (z. B. Chaudhri, 2007; Gulyás, 2011; Ingenhoff \& Koelling, 2012). Befragungen kamen nur selten vor (Gulyás 2009), auch wurden in wenigen Fällen Methoden kombiniert, wie u.a. von Altmeppen und Habisch (2008) oder von von Holly und Stark (2006), die ergänzend zur Inhaltsanalyse Experteninterviews führten, um über das Kommunizierte hinaus Kenntnisse über die Wahrnehmung von gesellschaftlicher Verantwortung zu erlangen.

Auf anderer Ebene liegt die Vorgehensweise von Sandoval (2014). Ihre Studie ist die einzig bekannte, die neben der „konstruierten“ Selbstpräsentation der gesellschaftlichen Verantwortung der Medienunternehmen in ihrer öffentlichen Kommunikation (Sandoval, 2014: 67) das Fremdbild bzw. Beschreibungen von „corporate watchdogs“ (Sandoval, 2014: 13) miteinbezog. Zwar konnten noch über zwei Dutzend Studien ausgemacht werden ${ }^{16}$ die sich mit CSR in der deutsch- und englischsprachigen (journalistischen) Berichterstattung beschäftigten, jedoch keine, die dies konkret in Bezug auf die Medienbranche tat. ${ }^{17}$

Im Zentrum der bisher durchgeführten Studien standen international tätige oder europäische Medienunternehmen. Häufig wurde aber auch ein engerer Zuschnitt gewählt. Je nach dem, ob generell die Größe (häufig gemessen am Umsatz) und/oder

16 Auf diese kann hier aus Platzmangel nicht eingegangen werden, doch konnten zahlreiche Erkenntnisse nutzbar gemacht werden, z. B. in Hinblick auf Suchworte und Auswahlkritierien der Artikel, die Datenbanken, aber auch in Bezug auf Erklärungen, warum CSR ein „schwieriges“ Thema für die Medien ist. Die deutschsprachigen Studien wurden als Referenzstudien für Teilstudie 3 herangezogen. Ferner liegen auch einige lateinamerikanische Studien vor, die jedoch nicht weiter berücksichtigt wurden.

17 Hier dürfen auch Fragen der Selbstthematisierung nicht außer Acht gelassen werden. Besonders soll auf die Arbeit von Pointner (2010) verwiesen werden und ihr „wirtschaftliches Distanz-Modell“ (Pointner, 2010: 85ff.), das auf die Aspekte der Selbst- und Konkurrenzbeobachtung aufmerksam macht. 
das Land die Auswahl bestimmte, finden sich manche Unternehmen in mehreren Studien wieder (wie z. B. Bertelsmann, Axel Springer oder Time Warner). Generell ist die Vergleichbarkeit der Ergebnisse aber als schwierig einzuschätzen, vor allem, da die Arbeiten mit unterschiedlichen theoretischen Fundamenten arbeiten und diese auf vielfältige Weise handhabbar für die Empirie machen (u. a. von Holly \& Stark, 2006; Chaudhri, 2007; Gulyás, 2011). Dies stellt für sich genommen bereits ein Ergebnis dar. Ebenso, dass sich die meisten Studien auf größere Unternehmen fokussierten, woran deutlich wird, dass hier Material vorhanden ist, das untersucht werden kann und über CSR öffentlich kommuniziert wird (und dies auf verschiedenen Kanälen). Es zeigt zudem, dass es noch vorwiegend die großen Unternehmen sind, die mit dem Thema sichtbar sind (häufig, da sie die entsprechenden Schlagworte benutzen) und prägend für die Branche („,role models“) oder sogar für die Wirtschaft an sich angesehen werden (je nach zugeschriebener Macht; Sandoval, 2014: 84).

Zusammenfassend kann festgehalten werden, dass Medienunternehmen ,einen bunten Mix an Projekten und Maßnahmen" im Bereich CSR durchführen (von Holly \& Stark, 2006: 64) und dass eine Entwicklung hin zu mehr Reporting stattgefunden hat (Gulyás, 2011) ${ }^{18}$ was die Relevanz des Themas für Medienunternehmen zeigt. Es fehlt jedoch häufig an der strategischen Ausrichtung und der professionellen Vorgehensweise (Trommershausen, 2011: 270). Weitere generelle Aussagen bleiben schwierig, auf der einen Seite, da eine große Breite von Medienunternehmen untersucht wurde und neben "klassischen“, die journalistische Inhalte erzeugen, auch solche einbezogen wurden, die eine breitere Definition des Begriffs Medienunternehmen voraussetzen. Weitere Schwierigkeiten bestehen andererseits darin, dass Unterschiede zwischen privaten und öffentlich-rechtlichen Unternehmen (Ingenhoff \& Koelling, 2012) wie auch länderspezifischen Unterschiede bestehen (Weder \& Karmasin, 2009).

Das Projekt, über das hier berichtet wird, hat sich auf deutsche Medienunternehmen konzentriert und mit einer Triangulation von Methoden (Befragung und Inhaltsanalysen) gearbeitet.

18 Der Begriff Reporting meint hier die unternehmensseitige Kommunikation über CSR, er wird in Abgrenzung zum Begriff Berichterstattung genutzt, der in Zusammenhang mit journalistischer Berichtertattung über CSR verwendet wird. 


\section{$3 \quad$ Methode und Instrumente}

Grundlage der empirischen Arbeit war eine bewusste Auswahl ${ }^{19}$, aufgrund derer fünf der größten zehn deutschen Medienunternehmen in die Analyse einbezogen wurden. ${ }^{20}$ Es handelte sich, basierend auf der Definition von Wirtz (2013), um „klassische“ Medienunternehmen. Berücksichtigt wurde ferner, dass private und öffentlich-rechtliche Unternehmen vertreten waren, um das duale Rundfunksystem abzubilden. ${ }^{21}$ Dem Sample gehörten die Axel Springer AG/SE, ${ }^{22}$ die Mediengruppe RTL Deutschland, die Verlagsgruppe Georg von Holtzbrinck sowie das ZDF und der Bayerische Rundfunk an. Zunächst wurden leitfadengestützte Experteninterviews mit den jeweils für CSR/Nachhaltigkeit verantwortlichen Akteuren in den Unternehmen geführt und qualitativ ausgewertet (dazu Meuser \& Nagel, 1991, 2009).

Im Bereich der öffentlichen Kommunikation wurden Pressemitteilungen als zentrales (Bertelsmann Stiftung, 2014: 62), aber wenig beachtetes Kommunikationsinstrument ausgewählt. Die im Zeitraum Januar 2011 bis Dezember 2013 veröffentlichten Texte wurden in einer quantitativen Inhaltsanalyse ausgewertet (Vollerhebung). Der Zugang zum Material erfolgte auf verschiedenen Wegen (Unternehmenswebsites/Pressearchive, presseportal.de sowie durch direkte Rückfrage bei den CSR-Verantwortlichen).

Im dritten Schritt wurde die journalistische Berichterstattung über die CSR-Aktivitäten der ausgewählten Medienunternehmen im gleichen Zeitraum inhalts-

19 Vorrecherchen zeigten, dass bereits die großen deutschen Medienunternehmen nicht alle einen Ansprechpartner für das Thema bennen konnten und einige zudem nicht extern über gesellschaftliche Verantwortung kommunizieren, was die möglichen Untersuchungsobjekte einschränkte und letztlich zu einer bewussten Auswahl von privaten und öffentlich-rechtlichen Medienunternehmen (definiert nach Wirtz, 2013) führte. Diese wurde letztlich auch durch die Teilnahmebereitschaft der Unternehmensvertreter beeinflusst.

20 „Formen der bewussten Auswahl“ werden „in der kommunikationswissenschaftlichen Forschung mit Abstand am häufigsten angewendet“, jedoch lassen sie, gerade bei derart kleinen Fallzahlen, „streng genommen keine statistisch repräsentativen Rückschlüsse von einer Stichprobe auf eine gegebene Grundgesamtheit" zu (Schmidt \& Zurstiege, 2007: 117 mit Verweis auf Gehrau \& Fretwurst, 2005, Möhring et al., 2005: 159; auch Brosius et al., 2009: 83ff.).

21 Auch öffentlich-rechtliche Unternehmen werden „als wirtschaftliche orientierte Unternehmen" (Altmeppen, 2010: 507) aufgefasst (auch Altmeppen \& Arnold, 2010: 332: „Medien waren immer erwerbswirtschaftliche Betriebe und selbst die öffentlich-rechtlichen Anstalten sind der Wirtschaftlichkeit verpflichtet").

22 Die Umwandlung von der AG in eine SE (Societas Europaea) fand Ende 2013, d.h. im Untersuchungszeitraum, statt. 
analytisch untersucht (Vollerhebung). Der Zugang erfolgte über verschiedenen Datenbanken (Lexis Nexis, Genios, WISO, DIGAS). ${ }^{23}$ Die Basis für die Auswahl der Pressemitteilungen und Artikel stellten Suchwortlisten dar, ${ }^{24}$ die zuvor durch die Recherche auf den Unternehmenswebsites und auf Grundlage der Unternehmensberichte sowie der Experteninterviews zusammengestellt wurden.

Die Instrumente (Leitfaden und Codebücher) fußten auf der in diesem Beitrag sehr komprimiert beschriebenen theoretischen Basis (CSR/CC-Literatur, gesellschaftliche Verantwortung in Verbindung mit der Strukturationstheorie, Medien und Journalismus als Organisationseinheiten).

Eine Auswahl zentraler Ergebnisse der fünf Leitfadeninterviews sowie der quantitativen Inhaltsanalysen der Pressemitteilungen $(\mathrm{N}=296)$ und der journalistischen Artikel ( $\mathrm{N}=303)$ werden im Folgenden zusammengefasst. Dies geschieht unter Rückgriff auf die Verantwortungsrelationen (Subjekt/Verantwortungsakteur - Objekt/Handlung - Adressat).

\section{$4 \quad$ Ausgewählte Ergebnisse}

296 Pressemitteilungen über CSR-/CC-Aktivitäten der ausgewählten Medienunternehmen wurden inhaltsanalytisch untersucht. Die meisten stammen vom ZDF (46,6 Prozent, N=296), darauf folgten Axel Springer (23,6 Prozent), der BR (15,2 Prozent) und RTL (14,5 Prozent). Von Holtzbrinck wurden im Untersuchungszeitraum keine Mitteilungen veröffentlicht, die von Relevanz waren. Die externe Kommunikation über gesellschaftliche Verantwortung von Holtzbrinck bestand zur Zeit der Untersuchung aus einer Seite in der Unternehmensbroschüre sowie der Website, auf der auf die von den Gesellschaftern und Geschäftsführern ehrenamtlich ausgeübte Tätigkeit hingewiesen, die „aktiv und/oder finanziell unterstützten Organisationen" aufgelistet sowie Hinweise auf verliehene Preise gegeben wurden (Verlagsgruppe Georg von Holtzbrinck GmbH, o. J.: 8). ${ }^{25}$

Diese Unterschiede weisen bereits auf unterschiedliche Kommunikationsstrategien hin, was in den Expertengesprächen bestätigt wurde. Wird von manchen (privatwirtschaftlichen) Unternehmen über alle CSR-Aktivitäten kommuniziert

23 Digitales Archiv der Axel Springer SE.

24 Zum Teil mussten die Listen aufgrund der unterschiedlichen Suchfunktionen und -möglichkeiten der Datenbanken je Archiv angepasst werden.

25 Die Leitfadengespräche wurden im Mai bis Juli 2013 geführt. Kurze Zeit später fand ein Relaunch der Website der Verlagsgruppe Georg von Holtzbrinck statt. 
(I1; I3) ${ }^{26}$, zum einen, weil das Unternehmen transparent und „nicht das große unbekannte Unternehmen“ sein will (I3), zum anderen aber auch, weil es sich um einen Bereich handelt, „womit man sich als Unternehmen natürlich gerne darstellt und gerne auch zeigt, dass man das macht" (I3). Die öffentlich-rechtlichen Unternehmen dagegen schätzen ihre externe Kommunikation eher als zurückhaltend ein (I5). Sie fände in „einem sehr überschaubaren Rahmen“ statt (I2), eine „gute Strategie“ sei hier "bescheiden aufzutreten“ (I2), was vor allem darauf zurückgeführt wird, dass öffentlich-rechtliche Unternehmen „sofort unter Druck“ geraten würden und leicht der Vorwurf aufkomme, fahrlässig mit Gebührengeldern umzugehen (I2). Auch wolle man den Eindruck vermeiden, dass die Projekte nur aus Imagegründen durchgeführt werden (I5). Gutes zu tun und nicht darüber zu reden, steht auch für Experte $4 \mathrm{im}$ Vordergrund. ${ }^{27} \mathrm{Ihm}$ geht es sehr stark um den guten, verantwortungsvollen Umgang im Unternehmen, was sich - wie auch die externen Engagements - von selbst im regionalen Umfeld verbreite (I4).

In den Interviews wurde ersichtlich, dass bei allen Unternehmen nicht über jede durchgeführte Maßnahme oder Initiative öffentlich informiert wird. Das Gefälle ist aber unterschiedlich groß. Zurückgeführt werden kann dies darauf, dass sehr viele kleinere Projekte und Aktivitäten durchgeführt werden, die unter CSR gefasst werden und auch darauf, wie CSR definiert wird. Die Experten erwähnen während der Gespräche eine Vielzahl von Aktivitäten, die von den Unternehmen nicht öffentlich kommuniziert wurden, z. B. Papierverbrauchsreduktionsmaßnahmen im Büroalltag, Heizen mit der Wärme von Studiolampen, Vorlesepaten, Schulungen für Kinder zum Umgang mit Medien, Umstellung der Fahrzeugflotte auf Elektrofahrzeuge, Mülltrennaktionen, Gesundheitsmanagement/-vorsorge usw.. Auch bestätigt sich, dass unterschiedliche Begriffe für diese Aktivitäten genutzt werden, sowohl intern als auch in der externen Kommunikation, wie Nachhaltigkeit, Verantwortung und Engagement, die englischen (CSR, CC, etc.) darunter aber kaum vertreten sind (was u. a. die Erweiterung der Suchwortlisten erforderte). Die Begründungen reichen von der generellen Ablehnung von Anglizismen (aus Gründen der Verständlichkeit; I3) bis hin zur Vermeidung von Begriffen, die „einen häufig sofort in ein Kästchenschema" bringen" (I2). Eigene konkret herausgearbeitete Definitionen werden fast nicht formuliert (Ausnahme: I3), sondern eher auf bestehende verwiesen (z. B. die der Europäischen Kommission, I2) oder breiter be- und umschrieben, was unter CSR verstanden wird (I4), oder aber betont, dass Begriffe und Definitionen

26 Die Interviews wurden anonymisiert und mit Fallnummern versehen, d.h. „II“ steht für Interview 1 usf..

27 Auf eine geschlechtsneutrale Formulierung wird aufgrund des Platzes und der Lesbarkeit verzichtet, gemeint sind beide Geschlechter. 
untergeordnete Bedeutung hätten, Taten seien das, was zähle (I1). Vieles laufe eher unstrukturiert ab, maßgeblich sei aber, dass etwas unternommen werde (I1). Ferner fällt auf, dass der Experte des familiengeführten Unternehmens sehr stark die Rolle der Unternehmenskultur, des Umgangs untereinander/mit den Mitarbeitern und die Aufgabe der Führungsperson, mit gutem Beispiel voranzugehen, betont („das ist meine Art der gelebten gesellschaftlichen Verantwortung“) (I4). Er spricht auch indirekt die ,Media Social Responsibility' (Altmeppen 2011a) an, dabei geht es für ihn, „um die Bereitschaft, Mittel einzusetzen (...) in der journalistischen Ausstattung" (I4).

Wer aber übernimmt für was in den analysierten Pressemitteilungen (PM) der Medienunternehmen Verantwortung? Ersichtlich wurde, dass in den PM des ZDF $(\mathrm{N}=200)$ das Unternehmen selbst am häufigsten als Verantwortungsträger vorkommt (44,0 Prozent) ${ }^{28}$ wie auch der BR in seinen PM mit 29,4 Prozent (N=68). Das ist nicht verwunderlich, da es sich um strategische Kommunikation handelt, es gilt aber nicht für alle Unternehmen: In Springers PM übernehmen vor allem Tochterunternehmen/spezielle Abteilungen/(etc.) Verantwortung (49,6 Prozent, $\mathrm{N}=113$ ), bei RTL sind es Prominente/Personen des öffentlichen Lebens, die vor allem als Verantwortungsträger genannt werden (33,8 Prozent, $\mathrm{N}=80)$, knapp vor (unternehmenseigenen) Stiftungen (32,5 Prozent).

Verantwortungsadressaten waren in erster Linie konkrete gesellschaftliche Gruppen (59,9 Prozent, N=444). ${ }^{29}$ Dies sind vor allem hilfsbedürftige Menschen (die z.B. durch Katastrophen oder die politische Lage in Not geraten sind; 26,8 Prozent, $\mathrm{N}=291^{30}$ ), aber auch Kinder und Jugendliche und Familien (17,5 Prozent) oder ehrenamtlich tätige Menschen (sogenannte „Helden des Alltags“; 13,4 Prozent). Ebenso wie junge Medienschaffende/Nachwuchs (13,4 Prozent) sowie Behinderte/ pflegebedürftige Menschen (6,2 Prozent), Mädchen/Frauen (3,8 Prozent), Schüler/ Lehrer (ohne Bildungseinrichtung; 9,3 Prozent) und Sonstige (z. B. Senioren, kulturschaffende Gruppen; 9,6 Prozent).

Interne Stakeholder spielen in den PM keine nennenswerte Rolle, obwohl in den Interviews von ausgeglichenem Engagement im Bereich der Binnen- und Außenwelt gesprochen wird (I2; I3). Aber einschränkend wird von einer öffentlich-rechtlichen

28 Hier stellen nicht mehr die Pressemitteilungen die Untersuchungseinheit dar, sondern die Nennungen der Verantwortungsträger (pro PM wurden der primäre und sekundäre erhoben, die hier zusammengefasst wurden), daher unterscheiden sich die nachfolgenden Zahlen von denen, die im Zusammenhang mit den PM allgemein genannt werden.

29 Auch hier wurden der primäre und der sekundäre Verantwortungsadressat zusammengefasst.

30 Es handelt sich hier um 291 und nicht 266 Nennungen, weil hier alle Adressaten der Medienunternehmen einflossen, auch wenn sie an späterer Stelle („tertiär“) vorkamen. 
Organisation auch angeführt, dass man sich seiner Verantwortung für die Mitarbeiter bewusst sei, jedoch gelte es, vorsichtig in der Kommunikation zu sein, damit es nicht heiße, „mit meinem Beitrag finanzieren die ihren Mitarbeitern Ferienbetreuung“, dies sei eine "ganz schmale Gratwanderung (...) darum brüsten wir uns in dem Falle nicht so speziell mit den Dingen, was wir unseren Mitarbeitern bieten“" (I5).

In Verbindung mit verantwortlichem Handeln werden vor allem journalistische Berichterstattung (wie Sondersendungen, Thementage; 34,9 Prozent; N=318), Wettbewerbe/Preise/Auszeichnungen (14,8 Prozent) und Workshops/Fortbildungen (12,6 Prozent) genannt.

Erfasst wurde darüber hinaus, ob Ressourcen genannt wurden. 93-mal wurden finanzielle Ressourcen erwähnt, am häufigsten von Springer. Dabei geht es zum Teil um erhebliche Summen (bis 205 Mio. Euro), die sich aber meist auf lange Zeiträume (bis 25 Jahre) beziehen. Auch personelle Ressourcen werden genannt, die in Zusammenhang mit Corporate Volunteering-Aktionen stehen, z. B. Unterstützung durch Mitarbeiter des BR bei den Special Olympics in München. In den CSR-Abteilungen/verantwortlichen Bereichen seien aber nur Mittel für das Gehalt, Reisen (I1) und/oder die "physische Umsetzung“, also z. B. für Druckkosten für den CSR-Bericht, vorhanden (I2), kein Budget für Aktivitäten. Durchführen und bezahlen müssten die Aktivitäten die jeweiligen Abteilungen selbst, alles andere wird von Experte 1 sehr stark als veraltet und ineffizient dargestellt. Neben dem Einsatz von finanziellen und personellen Ressourcen wurde insbesondere im Gespräch mit Experte 1 deutlich, welche Rolle autoritative Ressourcen spielen, bspw. sei die Rückendeckung der Geschäftsleitung bedeutend (I1).

Die Nähe zur Unternehmensführung äußert sich in der organisationalen Verankerung des Themas in allen untersuchten Unternehmen. Die Verantwortlichen sind entweder in direkten Stabstellen zum Vorstand zu finden (I1; I2, I5) oder es ist eine Ebene zwischengeschaltet. Viele Absprachen würden aber direkt erfolgen (I3) oder der Geschäftsführer kümmert sich selbst um das Thema (I4). In keinem Fall allerdings handelt es sich bei CSR um die einzige Aufgabe der Person, immer haben die Verantwortlichen noch weitere Aufgaben inne (I1-I5).

In der journalistischen Berichterstattung über die fünf ausgewählten Medienunternehmen und ihre Aktivitäten kommt der BR (und zugehörige Akteure) am häufigsten als (Verantwortungs-)Akteur vor (34,9 Prozent, $N=275),{ }^{31}$ gefolgt von Springer (32,0 Prozent), dem ZDF (13,1 Prozent), Holtzbrinck (10,9 Prozent) und RTL (9,1 Prozent).

31 Dies bezieht sich nur auf Medienunternehmen als Akteure (daneben werden noch weitere Akteure genannt, die bspw. als Koopertationspartner auftreten). 
In den deutlich meisten Fällen wird in den Artikeln dargestellt, dass von den Medienunternehmen Verantwortung wahrgenommen wird (81,3 Prozent, $\mathrm{N}=273$ ), ${ }^{32}$ in 18,7 Prozent wird Verantwortung vernachlässigt. Ein Ungleichgewicht zeigt sich auch in anderen Studien, die generell die deutschsprachige Berichterstattung über gesellschaftliche Verantwortung von Unternehmen untersucht haben (Stelzer 2009; Weder 2012), damit stellt dies zunächst kein medienunternehmenspezifisches Phänomen dar. Doch konnte dieses Ungleichgewicht dort mit den gewählten Aufgreifkriterien erklärt werden, da nur "positive" mit CSR in Verbindung bestehende Begriffe berücksichtigt wurden (Weder 2012: 183). Hier wurde auch die "negative“ Seite eingeschlossen; trotzdem sind es nicht die konfliktbeladenen oder negativen Themen, über die hauptsächlich berichtet wird, wie basierend auf der Nachrichtenwerttheorie hätte erwartet werden können (für einen Überblick Burkart 2002: 279ff.). Dies bedeutet entweder, dass es kaum Negatives zu berichten gibt oder aber nichts Negatives berichtet wird, weil Journalisten dann über die eigene Branche oder das eigene Unternehmen Schlechtes schreiben müssten. Tatsächlich lassen sich diesbezüglich Zusammenhänge feststellen: Beispielsweise wird im Hamburger Abendblatt am häufigsten (50 Prozent, $N=38$ ) über Axel Springers Verantwortungswahrnehmungen berichtet. Dies gilt auch für die WELT-Publikationen (56,3 Prozent, $\mathrm{N}=32$ ) oder für die Berliner Morgenpost (54,2 Prozent, $\mathrm{N}=24)$. Alle gehörten im Untersuchungszeitraum zum Springer-Konzern.

Der BR wird insgesamt am seltensten negativ erwähnt (nur 3 Nennungen, $N=51$ ), am häufigsten wird dies das ZDF (27,5 Prozent) und Springer (25,5 Prozent). Darunter sind vor allem Verantwortungsvernachlässigungen gegenüber Rezipienten (ZDF) und Mitarbeitern (Springer). Adressaten der Verantwortungswahrnehmungen sind, wie beim Selbstbild, vor allem konkrete gesellschaftliche Gruppen (z. B. beim BR in 74,3 Prozent, $N=148$ oder bei AS in 60,3 Prozent, $N=116$ ). Übernommen wird Verantwortung beim BR und Holtzbrinck am häufigsten durch Spenden (21 Prozent, $\mathrm{N}=81$ und 37,5 Prozent, $\mathrm{N}=16$ ), bei Springer durch Wettbewerbe/Preise/ Auszeichnungen (26,6 Prozent, N=79), beim ZDF durch das Ausstrahlen journalistischer Beiträge (33,3 Prozent, $N=15)$ und bei RTL durch den Einsatz „sonstiger betrieblicher Ressourcen" (30,8 Prozent, N=12). 92-mal wurden Angaben zu finanziellen Ressourcen gemacht. Die Werte streuen breit, aber auch hier werden hohe Summen erreicht. Personelle Ressourcen werden nur dreimal genannt, bspw. handelt es sich hierbei um eine Corporate Volunteering-Maßnahme von Springer. Darüber hinaus wurden noch sehr unterschiedliche „sonstige“ Ressourcen genannt.

32 In zwei Fällen konnte keine Zuordnung, ob CSR oder CSI, erfolgen. 


\section{$5 \quad$ Fazit}

Das Ziel des Forschungsprojektes war es, die CSR-Maßnahmen und -Instrumente in ausgewählten deutschen Medienunternehmen zu eruieren und deren Darstellung in der öffentlichen Kommunikation (Selbstbild) sowie das Fremdbild über die Unternehmen und ihre Aktivitäten in der journalistischen Berichterstattung empirisch zu untersuchen.

Die gesellschaftliche Verantwortung wurde zunächst von anderen Verantwortungsbereichen, die Medienunternehmen zugeschrieben werden, abgegrenzt und in Abstufungen in einer Matrix ganzheitlich von CSI bis CSR erfasst. Darauf aufbauend, sowie strukturiert durch die Verantwortungsrelationen (Subjekt, Handlungen, Objekt), konnte die gesellschaftliche Verantwortung von Medienunternehmen eingegrenzt und für die Inhaltsanalysen empirisch handhabbar gemacht werden.

Durch die Anwendung der Strukturationstheorie auf CSR wurden Regeln und Ressourcen identifiziert, deren Vorhandensein und Ausgestaltung in den Unternehmen analysiert werden konnte. Dabei war u. a. zentral, auf welche Regeln und Ressourcen sich die für CSR verantwortlichen Personen beziehen, welche demnach für sie handlungsleitend sind und folglich ihr Handeln ermöglichen, aber auch begrenzen. Der Einblick in die Ergebnisse zeigte, dass das Verständnis und der Ansatz der Unternehmen hinsichtlich CSR zum Teil stark voneinander abweichen, dies gilt auch für ihre Kommunikationsstrategien. Ersichtlich wurde auch, dass die genannten Verantwortungsakteure und auch die Verantwortungsadressaten unterschiedlich sind. Weiterhin kann festgehalten werden, dass sich die journalistische Berichterstattung hauptsächlich mit Verantwortungswahrnehmungen der Medienunternehmen beschäftigte (statt mit Verantwortungsvernachlässigungen).

Neben den detaillierten Ergebnissen, die Aufschluss über die Wahrnehmung und Kommunikation der ausgewählten Medienunternehmen geben und aufzeigen, welches Fremdbild in der journalistischen Berichterstattung geschaffen wird, bieten die CSI-CSR-Matrix und die Anwendung der Strukturationstheorie auf CSR einen weiteren Mehrwert, da diese Frameworks nicht nur auf Medienunternehmen, sondern auch auf andere Branchen übertragbar sind. Denkbar ist dadurch die Möglichkeit der Vergleichbarkeit von Ergebnissen von Unternehmen aus verschiedenen Feldern. 


\section{Literatur}

Altmeppen, K.-D. (2006). Journalismus und Medien als Organisationen. Leistungen, Strukturen und Management. Wiesbaden: VS Verlag.

Altmeppen, K.-D. (2008a). Die soziale Verantwortung des Journalismus. Communicatio Socialis, 41(3): 241-253.

Altmeppen, K.-D. (2008b). Diffuse Geschäftsgrundlagen. Die schwierige Beziehung von Journalismus und Medien. In: B. Pörksen, W. Loosen \& A. Scholl (Hrsg.), Paradoxien des Journalismus. Theorie - Empirie - Praxis. Festschrift für Siegfried Weischenberg (pp. 81-99). Wiesbaden: VS Verlag.

Altmeppen, K.-D. (2008c). Ökonomisierung der Medienunternehmen: Gesellschaftlicher Trend und sektorspezifischer Sonderfall. In: A. Maurer \& U. Schimank (Hrsg.), Die Gesellschaft der Unternehmen - Die Unternehmen der Gesellschaft. Gesellschaftstheoretische Zugänge zum Wirtschaftsgeschehen (pp. 237-251). Wiesbaden: VS Verlag.

Altmeppen, K.-D. (2010). Journalistische Beobachter in der öffentlichen (Verantwortungs-) Kommunikation. In: H. Backhaus-Maul, C. Biedermann, S. Nährlich \& J. Polterauer (Hrsg.), Corporate Citizenship in Deutschland. Gesellschaftliches Engagement von Unternehmen. Bilanz und Perspektiven. 2. Aufl. (pp. 497-508). Wiesbaden: VS Verlag für Sozialwissenschaften.

Altmeppen, K.-D. (2011a). Journalistische Berichterstattung und Media Social Responsibility: Über die doppelte Verantwortung von Medienunternehmen. In: J. Raupp, S. Jarolimek \& F. Schultz (Hrsg.), Handbuch CSR. Kommunikationswissenschaftliche Grundlagen, disziplinäre Zugänge und methodische Herausforderungen. Mit Glossar (pp. 247-268). Wiesbaden: VS Verlag.

Altmeppen, K.-D. (2011b). Medienökonomisch handeln in der Mediengesellschaft. Eine Mikro-Meso-Makro-Skizze anhand der Ökonomisierung der Medien. In: T. Quandt \& B. Scheufele (Hrsg.), Ebenen der Kommunikation. Mikro-Meso-Makro-Links in der Kommunikationswissenschaft (pp. 233-258). Wiesbaden: VS Verlag.

Altmeppen, K.-D., \& Arnold, K. (2010). Ethik und Profit. In: C. Schicha \& C. Brosda (Hrsg.), Handbuch Medienethik (pp. 331-347). Wiesbaden: VS Verlag.

Altmeppen, K.-D. \& Habisch, A. (2008). CSR in den Medien. Eine Inhaltsanalyse deutscher Printmedien und Experteninterviews. Eichstätt: Unveröffentlicher Forschungsbericht.

Altmeppen, K.-D. \& Bracker, I. (im Druck). Gesellschaftliche Verantwortung von Unternehmen in Deutschland aus Perspektive der Kommunikationswissenschaft. In: H. Backhaus-Maul, M. Kunze, \& S. Nährlich (Hrsg.), Gesellschaftliche Verantwortung von Unternehmen in Deutschland. Wiesbaden: VS Verlag.

Axel Springer SE. (2015). Verantwortung und Nachhaltigkeit. http://www.axelspringer. de/artikel/Verantwortung-und-Nachhaltigkeit_40542.html. Zugegriffen: 05.03.2015.

Becker, A. (1996). Rationalität strategischer Entscheidungsprozesse. Ein strukturationstheoretisches Konzept. Wiesbaden: Deutscher Universitäts-Verlag.

Bertelsmann Stiftung. (2014). CRI Corporate Responsibility Index 2013. Erfolgsfaktoren unternehmerischer Verantwortung. http://www.bertelsmann-stiftung.de/de/publikationen/publikationen/publikation/did/cri-corporate-responsibility-index-2013/. Zugegriffen: 19.11.2014.

Bluhm, K. (2008). Corporate Social Responsibility - Zur Moralisierung von Unternehmen aus soziologischer Perspektive. In: A. Maurer \& U. Schimank (Hrsg.), Die Gesellschaft der 
Unternehmen - Die Unternehmen der Gesellschaft. Gesellschaftstheoretische Zugänge zum Wirtschaftsgeschehen (pp. 144-162). Wiesbaden: VS Verlag.

Bowen, H. R. (1953). Social Responsibilities of the Businessman. New York: Harper \& Brothers. Bracker, I., Schuhknecht, S. \& Altmeppen, K.-D. (im Druck). Managing values: An approach to analyze CSR in media companies from structuration theory perspective. In: K.-D. Altmeppen, C. A. Hollifield \& J. van Loon (Hrsg.), Value-oriented media management. Decision making between profit and responsibility. Wiesbaden: VS Verlag.

Brosius, H.-B., Koschel, F. \& Haas, A. (2009). Methoden der empirischen Kommunikationsforschung. Eine Einführung. 5. Aufl. Wiesbaden: VS Verlag für Sozialwissenschaften.

Burkart, R. (2002). Kommunikationswissenschaft. Grundlagen und Problemfelder. Umrisse einer interdisziplinären Sozialwissenschaft. 4., überarbeitete und aktualisierte Aufl. Wien: Böhlau Verlag.

Carroll, A. B. (1979). A Three-Dimensional Conceptual Model of Corporate Performance. Academy of Management Review, 4(4): 497-505.

Carroll, A. B. (1991). The Pyramid of Corporate Social Responsibility: Toward the Moral Managament of Organizational Stakeholders. Business Horizons, 34(4): 39-48.

Carroll, A. B. (1999). Corporate Social Responsibility. Evolution of a Definitional Construct. Business \& Society, 38(3): 268-295.

Chaudhri, V. (2007). Corporate Social Responsibility and the Media: What the Big Five media corporations are communicating about their social responsibilities. National Communication Association. Chicago.

Clark, T. S. \& Grantham, K. N. (2012). What CSR is not: Corporate Social Irresponsibility. In: R. Tench, W. Sun \& B. Jones (Hrsg.), Corporate Social Irresponsibility: A Challenging Concept (pp. 23-41). Bingley: Emerald Group Publishing Limited.

Galonska, C. (2012). Die Wirtschaftselite im gesellschaftlichen Abseits. Von der Klasse an sich zur Klasse für sich? Wiesbaden: Springer VS.

Galonska, C., Imbusch, P. \& Rucht, D. (2007). Einleitung: Die gesellschaftliche Verantwortung der Wirtschaft. In: P. Imbusch \& D. Rucht (Hrsg.), Profit oder Gemeinwohl? Fallstudien zur gesellschaftlichen Verantwortung von Wirtschaftseliten (pp. 9-29). Wiesbaden: VS Verlag.

Garriga, E. \& Melé, D. (2004). Corporate Social Responsibility Theories: Mapping the Territory. Journal of Business Ethics, 53(1-2): 51-71.

Gehrau, V. \& Fretwurst, B. (2005). Auswahlverfahren in der Kommunikationswissenschaft. Ein Untersuchung aktueller Veröffentlichungen über empirische Studien in der Kommunikationswissenschaft. In: V. Gehrau, B. Fretwurst, B. Krause \& G. Daschmann (Hrsg.), Auswahlverfahren in der Kommunikationswissenschaft (pp. 13-31). Köln: Herbert von Halem.

Giddens, A. (1997). Die Konstitution der Gesellschaft. 3. Aufl. Frankfurt: Campus.

Grayson, D. (2009). Corporate Responsibility und die Medien. Wie die Medien über gesellschaftliche Verantwortung von Unternehmen berichten und wie sie ihrer eigenen Verantwortung als Unternehmen nachkommen. http://www.cccdeutschland.org/sites/ default/files/CCCDebatte_02_dt.pdf. Zugegriffen: 14.09.2012.

Grunwald, A. \& Kopfmüller, J. (2012). Nachhaltigkeit. 2., aktualisierte Auflage. Frankfurt: Campus Verlag.

Gulyás, Á. (2009). Corporate social responsibility in the British media industries - preliminary findings. Media, Culture \& Society, 31(4): 657-668.

Gulyás, Á. (2011). Demons into Angels? Corporate Social Responsibility and Media Organisations. Critical Survey, 23(2): 56-74. 
Hauff, V. (Hrsg.) (1987). Unsere gemeinsame Zukunft. Der Brundtland-Bericht der Weltkommission für Umwelt und Entwicklung. Greven: Eggenkamp Verlag.

Heinrich, J. (2010). Medienökonomie: Band 1: Mediensystem, Zeitung, Zeitschrift, Anzeigenblatt. 3. Aufl. Wiesbaden: VS Verlag.

Hiß, S. (2007). Corporate Social Responsibility. Über die Durchsetzung von Stakeholder-Interessen im Shareholder-Kapitalismus. Berliner Debatte Initital, 18(4/5): 6-15.

Hohlfeld, R. (2002). Distinktionsversuche im Fernsehjournalismus. Das Verschwinden von Journalismus durch Inszenierung. In: A. Baum \& S. J. Schmidt (Hrsg.), Fakten und Fiktion: Über den Umgang mit Medienwirklichkeiten (pp. 101-113). Konstanz: UVK Verlagsgesellschaft.

von Holly, I. \& Stark, B. (2006). Gesellschaftliches Engagement deutscher Medienverlage. CSR-Strategien auf dem Prüfstand. prmagazin, 37(12): 61-68.

Hülsewiesche, V. M. (2010). Corporate Social Responsibility. Unter besonderer Berücksichtigung der Verantwortung von Medienunternehmen am Beispiel der WAZ Mediengruppe. Masterarbeit, Ruhr-Universität Bochum, Fakultät für Sozialwissenschaft. http://www. sowi.rub.de/mam/content/heinze/weitere/masterarbeit_h__lsewiesche.pdf.ZZugegriffen: 01.06.2012.

Imbusch, P. \& Rucht, D. (Hrsg.) (2007). Profit oder Gemeinwohl? Fallstudien zur gesellschaftlichen Verantwortung von Wirtschaftseliten. Wiesbaden: VS Verlag.

Ingenhoff, D. \& Koelling, A. M. (2012). Media governance and corporate social responsibility of media organizations: an international comparison. Business Ethics: A European Review, 21(2): 154-167.

Jarolimek, S. (2012). CSR-Kommunikation. Begriff, Forschungsstand und methodologische Herausforderungen. uwf - UmweltWirtschaftsForum, 19(3-4): 135-141.

Jarolimek, S. \& Raupp, J. (2011). Verantwortung und Nachhaltigkeit in Theorie und Empirie. Eine Synopse des Forschungsstands und Anschlussmöglichkeiten für die Kommunikationswissenschaft. Medien Journal, 35(1): 16-29.

Jonker, J., Stark, W. \& Tewes, S. (2011). Corporate Social Responsibility und nachhaltige Entwicklung. Einführung, Strategie und Glossar. Berlin: Springer-Verlag.

Karmasin, M. \& Weder, F. (2008). Organisationskommunikation und CSR: Neue Herausforderungen an Kommunikationsmanagement und PR. Wien: Lit Verlag.

Karmasin, M. \& Weder, F. (2009). Verantwortung von, in und durch Medien. uwf - UmweltWirtschaftsForum, 17(1): 45-50.

Klare, J. (2010). Kommunikationsmanagement deutscher Unternehmen in China. Eine strukturationstheoretische Analyse internationaler PR. Wiesbaden: VS Verlag.

Kleinsteuber, H. J. \& Thomaß, B. (2004). Medienökonomie, Medienkonzerne und Konzentrationskontrolle. In: K.-D. Altmeppen \& M. Karmasin (Hrsg.), Medien und Ökonomie. Band 2: Problemfelder der Medienökonomie (pp. 121-158). Wiesbaden: VS Verlag.

Lamla, J. (2003). Anthony Giddens. Frankfurt am Main: Campus Verlag.

Loew, T., Ankele, K., Braun, S. \& Clausen, J. (2004). Bedeutung der internationalen CSR Diskussion für Nachhaltigkeit und die sich daraus ergebenden Anforderungen an Unternehmen mit Fokus Berichterstattung. Endbericht an das Bundesministerium für Umwelt, Naturschutz und Reaktorsicherheit Geschäftszeichen GI2 - 46043/136, Münster und Berlin.

Möhring, W., Tiele, A., Scherer, H. \& Schneider, B. (2005). Repräsentative Stichprobe des Zeitungsangebotes - die Methode der publizistischen Stichprobe als computerbasiertes 
Auswahlverfahren. In: V. Gehrau, B. Fretwurst, B. Krause \& G. Daschmann (Hrsg.), Auswahlverfahren in der Kommunikationswissenschaft (pp. 158-172). Köln: Halem Verlag.

Maring, M. (2001). Kollektive und korporative Verantwortung. Begriffs- und Fallstudien aus Wirtschaft, Technik und Alltag. Münster: LIT.

Mast, C. \& Stehle, H. (2009). Corporate Social Responsibility - Modeerscheinung oder mehr? In: S. J. Schmidt \& J. Tropp (Hrsg.), Die Moral der Unternehmenskommunikation: Lohnt es sich, gut zu sein? (pp. 170-186). Köln: Halem Verlag.

Meuser, M. \& Nagel, U. (1991). ExpertInneninterviews - vielfach erprobt, wenig bedacht. Ein Beitrag zur qualitativen Methodendiskussion. In: D. Garz \& K. Krainer (Hrsg.), Qualitativ-empirische Sozialforschung. Konzepte, Methoden, Analysen (pp. 441-471). Opladen: Westdeutscher Verlag.

Meuser, M. \& Nagel, U. (2009). Experteninterview und der Wandel der Wissensproduktion. In: A. Bogner, B. Littig \& W. Menz (Hrsg.), Experteninterviews: Theorien, Methoden, Anwendungsfelder. 3., überarbeitete Aufl. (pp. 35-60). Wiesbaden: VS Verlag.

Ortmann, G., Windeler, A., Becker, A. \& Schulz, H.-J. (1990). Computer und Macht in Organisationen. Mikropolitische Analysen. Opladen: Westdeutscher Verlag.

Palazzo, G. (2009). Der aktuelle Stand der internationalen wissenschaftlichen Forschung zur Corporate Social Responsibility (CSR). Gutachten für das Bundesministerium für Arbeit und Soziales. Universität Lausanne. https:/www.csr-in-deutschland.de/fileadmin/ user_upload/Downloads/CSR_in_Deutschland/CSR_Forum/AG_3_Expertise_zur_Betriebswirtschaftslehre_aus_internationaler_Perspektive.pdf. Zugegriffen: 02.03.2015.

Pointner, N. (2010). In den Fängen der Ökonomie? Ein kritischer Blick auf die Berichterstattung über Medienunternehmen in der deutschen Tagespresse. Wiesbaden: VS Verlag.

Röttger, U. (2005). Kommunikationsmanagement in der Dualität von Struktur. Die Strukturationstheorie als kommunikationswissenschaftliche Basistheorie. Medienwissenschaft Schweiz, 1(2): 12-19.

Röttger, U. (2010). Public Relations. Organisation und Profession. 2. Aufl. Wiesbaden: VS Verlag.

Raupp, J., Jarolimek, S. \& Schultz, F. (2011). Corporate Social Responsibility als Gegenstand der Kommunikationsforschung. Einleitende Anmerkungen, Definitionen und disziplinäre Perspektiven. In: J. Raupp, S. Jarolimek \& F. Schultz (Hrsg.), Handbuch CSR. Kommunikationswissenschaftliche Grundlagen, disziplinäre Zugänge und methodische Herausforderungen. Mit Glossar (pp. 9-18). Wiesbaden: VS Verlag.

Sandoval, M. (2014). From Corporate to Social Media. Critical perspectives on corporate social responsibility in media and communciation industries. New York: Routledge.

Schaltegger, S. (2011). Von CSR zu Corporate Sustainability. In: B. Sandberg \& K. Lederer (Hrsg.), Corporate Social Responsibility in kommunalen Unternehmen (pp. 187-199). Wiesbaden: VS Verlag.

Schmidpeter, R. (2012). Unternehmerische Verantwortung - Hinführung und Überblick über das Buch. In: A. Schneider \& R. Schmidpeter (Hrsg.), Corporate Social Responsibility. Unternehmensführung in Theorie und Praxis (pp. 1-13). Berlin: Springer-Verlag. Schmidt, S. J. \& Zurstiege, G. (2007). Kommunikationswissenschaft. Systematik und Ziele. Reinbek bei Hamburg: Rowohlt Verlag.

Schneider, A. (2012). Reifegradmodell CSR - eine Begriffsklärung und -abgrenzung. In: A. Schneider \& R. Schmidpeter (Hrsg.), Corporate Social Responsibility. Unternehmensführung in Theorie und Praxis (pp. 17-38). Berlin: Springer-Verlag. 
Schneidewind, U. (1998). Die Unternehmung als strukturpolitischer Akteur. Kooperatives Schnittmengenmanagement im ökologischen Kontext. Marburg: Metropolis-Verlag.

Schwarz, S. (2008). Strukturation, Organisation und Wissen: Neue Perspektiven in der Organisationsberatung. Wiesbaden: VS Verlag.

Stelzer, T. (2009). Corporate Social Responsibility in der Berichterstattung deutscher Tageszeitungen. Berlin: Polisphere.

Tench, R., Sun, W. \& Jones, B. (2012). Corporate Social Irresponsibility: A Challenging Concept. Bingley: Emerald Group Publishing.

Toennesen, C., Hodgson: \& Mimmack, F. (2013). Mirrors or Movers? Framing the debate about the impact of media content. http://mirrorsormovers.com/_media/documents/ report.pdf. Zugegriffen: 21.02.2014.

Trommershausen, A. (2011). Corporate Responsibility in Medienunternehmen. Köln: Herbert von Halem Verlag.

Verlagsgruppe Georg von Holtzbrinck GmbH. (o. J.). Verlagsgruppe Georg von Holtzbrinck. Stuttgart.

Weber, B. \& Rager, G. (2006). Medienunternehmen - Die Player auf den Medienmärkten. In: C. Scholz (Hrsg.), Handbuch Medienmanagement (pp. 117-143). Berlin: Springer-Verlag.

Weder, F. (2012). Die CSR-Debatte in den Printmedien. Anlässe, Themen, Deutungen. Wien: Facultas. wuv.

Weder, F. \& Karmasin, M. (2009). Österreichische Medienunternehmen in der Verantwortung: Selbstregulierung als Antwort auf die Frage nach der gesellschaftlichen Verantwortung (CSR) von Medienunternehmen. In: B. Stark \& M. Magin (Hrsg.), Die österreichische Medienlandschaft im Umbruch. Band 3 (pp. 321-346). Wien: Österreichische Akademie der Wissenschaften.

Werner, M. H. (2006). Verantwortung. In: M. Düwell, C. Hübenthal \& M. H. Werner (Hrsg.), Handbuch Ethik. 2., aktualisierte und erw. Aufl. (pp. 541-548). Stuttgart: J.B. Metzler.

Wirtz, B. W. (2013). Medien- und Internetmanagement. 8., aktualisierte und überarb. Aufl. Wiesbaden: Springer Gabler.

Zerfaß, A. (1996). Unternehmensführung und Öffentlichkeitsarbeit. Grundlegung einer Theorie der Unternehmenskommunikation und Public Relations. Opladen: Westdeutscher Verlag.

Zyglidopoulos, C., Georgiadis, A. P., Carroll, C. E. \& Siegel, D. S. (2012). Does media attention drive corporate social responsibility? Journal of Business Research, 65(11): 1622-1627. 
Open Access Dieses Kapitel wird unter der Creative Commons Namensnennung - Nicht kommerziell 2.5 International Lizenz (http://creativecommons.org/licenses/by-nc/2.5/deed. de) veröffentlicht, welche für nicht kommerzielle Zwecke die Nutzung, Verbreitung und Wiedergabe in jeglichem Medium und Format erlaubt, sofern Sie den/die ursprünglichen Autor(en), den Titel des Werks und die Quelle ordnungsgemäß nennen, einen Link zur Creative Commons Lizenz beifügen und im Falle einer Abwandlung durch einen entsprechenden Hinweis deutlich erkennbar machen, dass Änderungen vorgenommen wurden.

Die in diesem Kapitel enthaltenen Bilder und sonstiges Drittmaterial unterliegen ebenfalls der genannten Creative Commons Lizenz, sofern sich aus der Abbildungslegende nichts anderes ergibt. Sofern das betreffende Material nicht unter der genannten Creative Commons Lizenz steht und die betreffende Handlung nicht nach gesetzlichen Vorschriften erlaubt ist, ist auch für die oben aufgeführten nicht-kommerziellen Weiterverwendungen des Materials die Einwilligung des jeweiligen Rechteinhabers einzuholen. 